\title{
Microstructure and Mechanical Properties of Zircaloy-4 Cladding Hydrogenated at Temperatures Typical for Loss-of-Coolant Accident (LOCA) Conditions
}

\section{A. Pshenichnikov, J. Stuckert, M. Walter}

anton.pshenichnikov@kit.edu, juri.stuckert@kit.edu, mario.walter@kit.edu
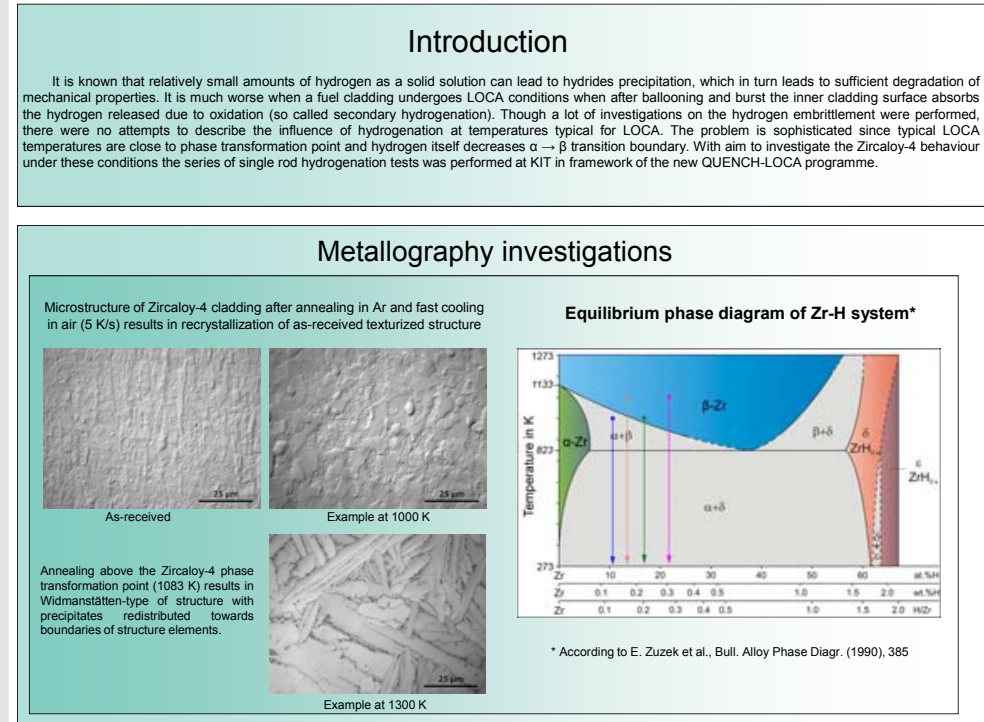

Scanning Electron Microscopy

Grinded and polished specimen

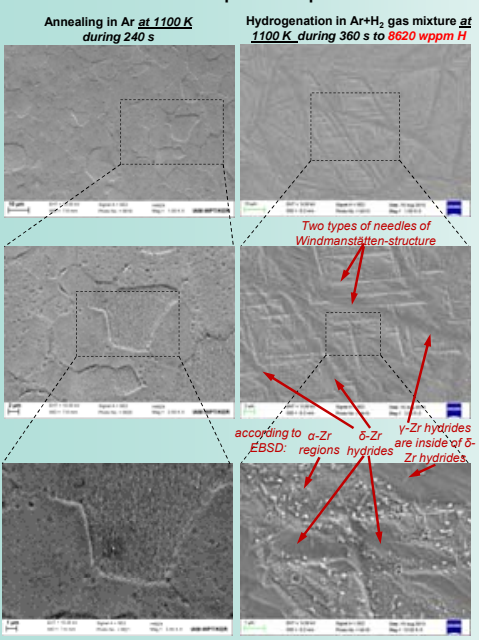

Fractured surface after tensile tests

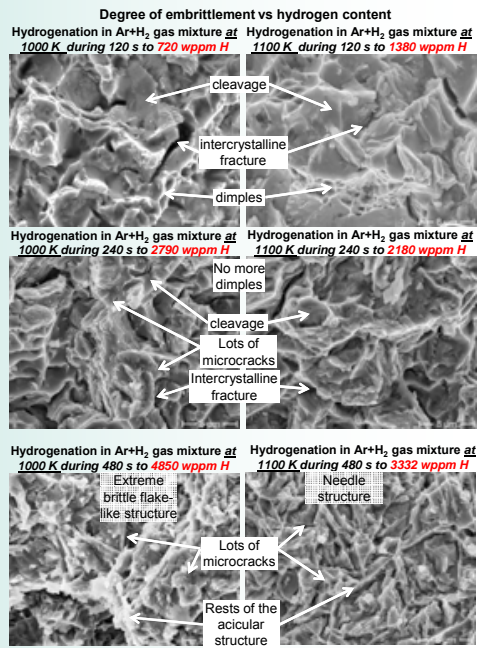

The as-received sample was highy texturized. During the heat treatment in absence of hydrogen the usual proesss or Hydrogen changes the appearance of microstructural pattern cardinally.
and a-rr clusters will be developed. During the cooling phase of hydrogenate
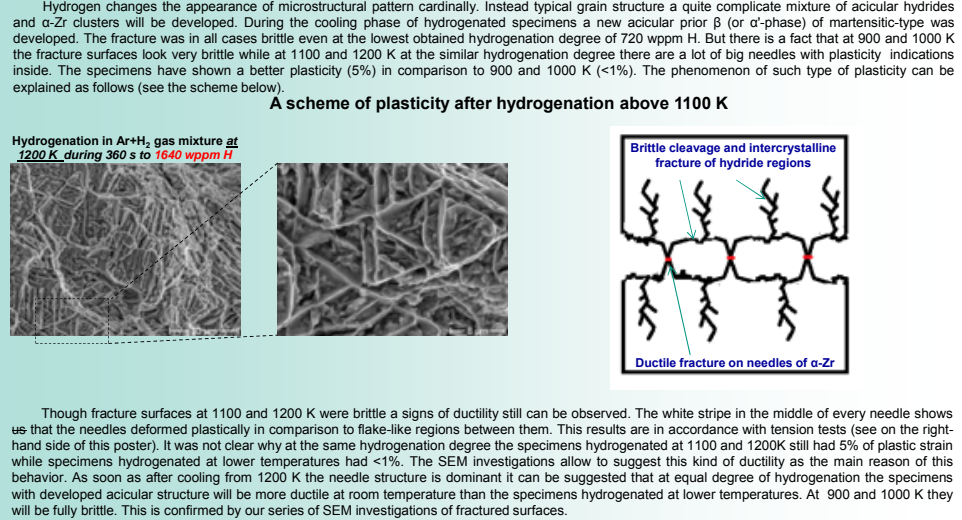

Tensile test at room temperature

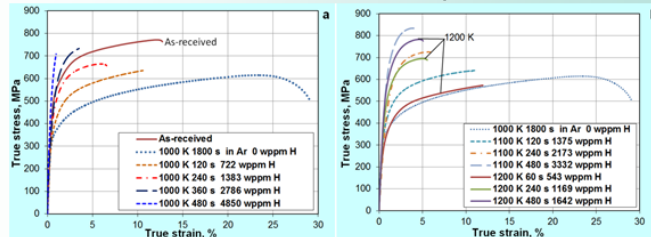

Microhardness test
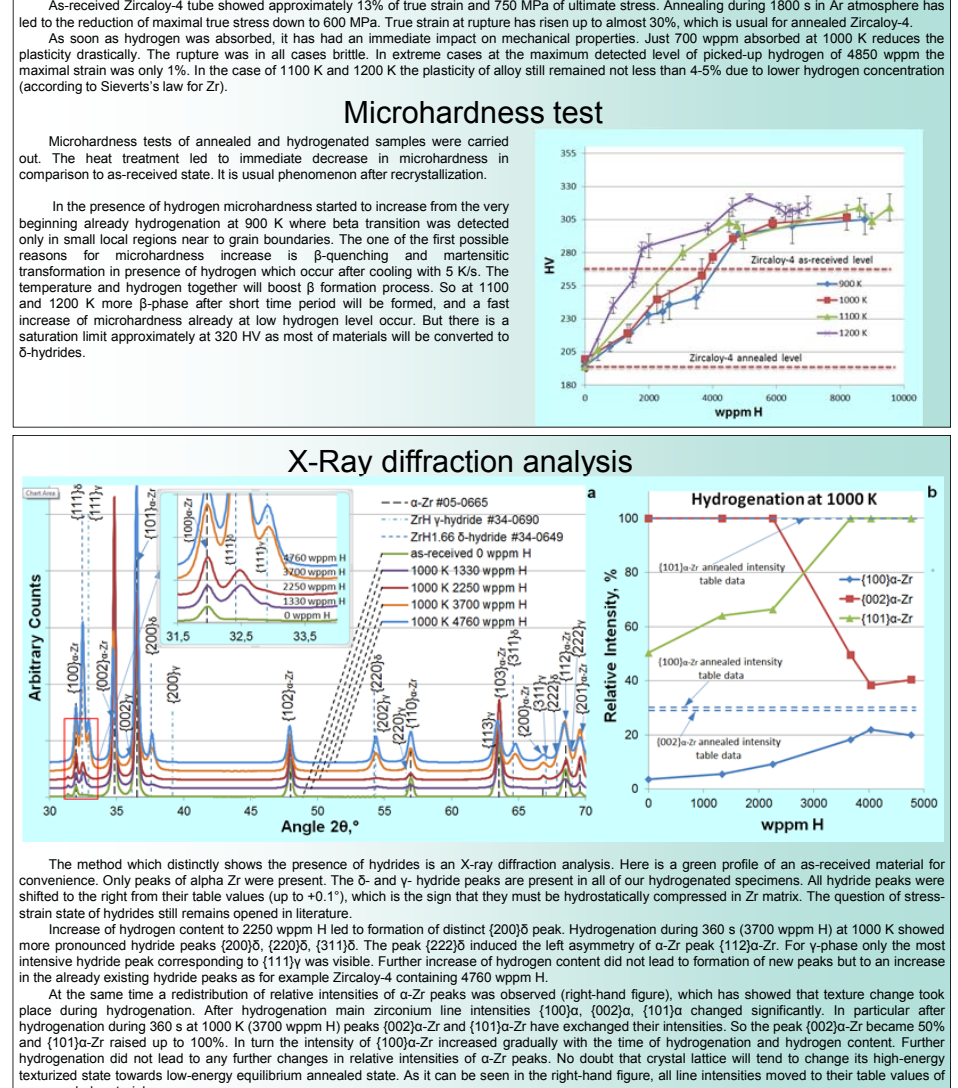

Hexagonal lattice parameter analysis

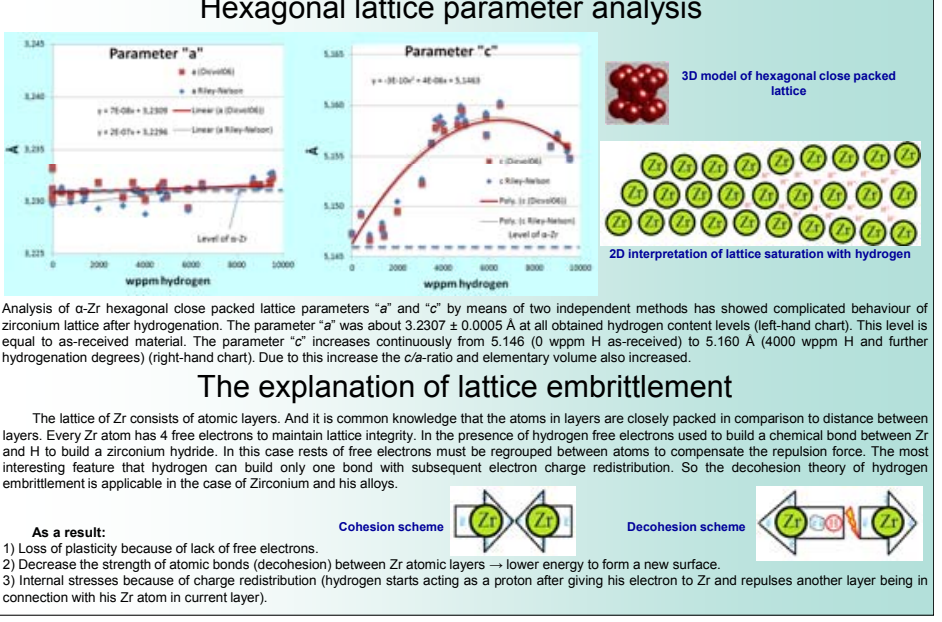

$$
\text { (2). (20) }
$$

(1) 0 (2)

\section{Conclusions} analysis showed that even at lowest obtained hydrogen content the fracture was of mixed type with signs of plasticity. With increasing

Microhardness tests showed a relationship between hydrogen hardening, annealing softening and hardening due to

lattice which is indicated by increase of the lattice parameter " $c$.

Because carried out observations have proved only the presence of hydrides and gave
morphology and orientation of hydrides and to separate them from other structural features.

The performed experinc

The proposed scheme of the decohesion mechanism helps to understand the embrittlement of zirconiu 